\title{
Schwinger-Fronsdal Theory of Abelian Tensor Gauge Fields
}

\author{
Sebastian GUTTENBERG and George SAVVIDY \\ Institute of Nuclear Physics, Demokritos National Research Center, \\ Agia Paraskevi, GR-15310 Athens, Greece \\ E-mail: guttenb@inp.demokritos.gr,savvidy@inp.demokritos.gr \\ URL: http://hep.itp.tuwien.ac.at/ basti/,http://www.inp.demokritos.gr/ savvidy/
}

Received April 23, 2008, in final form September 01, 2008; Published online September 04, 2008

Original article is available at http://www.emis.de/journals/SIGMA/2008/061/

\begin{abstract}
This review is devoted to the Schwinger and Fronsdal theory of Abelian tensor gauge fields. The theory describes the propagation of free massless gauge bosons of integer helicities and their interaction with external currents. Self-consistency of its equations requires only the traceless part of the current divergence to vanish. The essence of the theory is given by the fact that this weaker current conservation is enough to guarantee the unitarity of the theory. Physically this means that only waves with transverse polarizations are propagating very far from the sources. The question whether such currents exist should be answered by a fully interacting theory. We also suggest an equivalent representation of the corresponding action.
\end{abstract}

Key words: Abelian gauge fields; Abelian tensor gauge fields; high spin fields; conserved currents; weakly conserved currents

2000 Mathematics Subject Classification: 81T10; 81T13; 70S05; 70S10; 70S15; 35L05; 35L10

\section{Introduction}

We shall start from the formulation of the Schwinger-Fronsdal action for symmetric Abelian tensor gauge field of rank $s, A_{\lambda_{1} \ldots \lambda_{s}}[1,2,3]$. The development which leads to the discovery of this action and the corresponding review articles can be found in the extended literature $[6,7,8,9,10,11,12,13,14,15,16,17,23,24,25]$. The theory is gauge invariant, but to our best knowledge there is no unique and systematic way to extend this action to an interacting theory from some sort of gauge principle. This is in contrast with the Yang-Mills theory, where one can formulate the gauge principle, to derive transformation properties of the vector gauge field and to find out the corresponding gauge invariant action.

Therefore we shall postulate the quadratic form for the Lagrangian $\mathcal{L}$ and then describe its invariant and physical properties. The variation of the Schwinger-Fronsdal action allows to derive the equation of motion for a symmetric Abelian tensor gauge field of rank $s, A_{\lambda_{1} \ldots \lambda_{s}}$, in the presence of an external current $J_{\lambda_{1} \ldots \lambda_{s}}$

$$
(L A)_{\lambda_{1} \ldots \lambda_{s}}=J_{\lambda_{1} \ldots \lambda_{s}}
$$

where $L$ is a linear differential operator of second order. As we shall see, the equation describes the propagation of transverse polarizations of a spin- $s$ gauge boson and its interaction with the external current. Self-consistency of this equation requires that the traceless part ${ }^{1}$ of the current divergence should vanish $[1,2]$

$$
\partial^{\mu} J_{\mu \lambda_{2} \ldots \lambda_{s}}-\frac{1}{d+2 s-6} \sum_{2} \eta_{\lambda_{2} \lambda_{3}} \partial^{\mu} J_{\mu \lambda_{4} \ldots \lambda_{s}}^{\prime}=0 .
$$

\footnotetext{
${ }^{1}$ Fields and currents are double traceless, see Section 2 and especially footnote 5.
} 
This is a weaker conservation law of the current, if one compares it with the fully conserved current $\partial^{\mu} J_{\mu \lambda_{2} \ldots \lambda_{s}}=0$. The weaker current conservation law nevertheless guarantees the unitarity of the theory $[1,2]$. Physically this means that only waves with transverse polarizations are propagating very far from the sources, as it is the case for fully conserved currents [4]. It is outside of the scope of this theory to answer the question if such currents exist or not. It should be answered by a fully interacting theory. At the end of this review we shall also suggest an equivalent representation of the corresponding action.

The subject which we do not touch in this review is the question of possible extension of this theory to a fully interacting theory. The answer still remains uncertain, but self-consistency and beauty of this theory tell us that probably some part of it may become essential in the construction of an interacting theory $[12,13]$.

For the recent development of interacting gauge field theories based on the extension of the gauge principle to non-Abelian tensor gauge fields see references [27, 28, 29, 30] and for the calculation of the production cross section of spin-two non-Abelian tensor gauge bosons see [33]. The interacting field theories in anti-de Sitter space-time background are reviewed in $[18,19,20,21,22]$.

\section{Schwinger-Fronsdal action}

The Schwinger-Fronsdal action for symmetric Abelian tensor gauge fields of rank s was derived first for the rank-3 gauge field by Schwinger in [1] and then was generalized by Fronsdal [2] to arbitrary symmetric rank-s field exploring the massless limit of the Singh-Hagen action for massive tensor fields [3]. The massless action has the following form

$$
\begin{aligned}
S[A]= & \int d x^{d} \frac{1}{2} \partial_{\mu} A_{\lambda_{1} \ldots \lambda_{s}} \partial^{\mu} A^{\lambda_{1} \ldots \lambda_{s}}-\frac{s}{2} \partial_{\mu} A^{\mu \lambda_{2} \ldots \lambda_{s}} \partial^{\nu} A_{\nu \lambda_{2} \ldots \lambda_{s}} \\
& -\frac{s(s-1)}{2} A_{\lambda_{3} \ldots \lambda_{s}}^{\prime} \partial_{\mu} \partial_{\nu} A^{\mu \nu \lambda_{3} \ldots \lambda_{s}}-\frac{s(s-1)}{4} \partial_{\mu} A^{\prime}{ }_{\lambda_{3} \ldots \lambda_{s}} \partial^{\mu} A^{\prime \lambda_{3} \ldots \lambda_{s}} \\
& -\frac{s(s-1)(s-2)}{8} \partial_{\mu} A^{\prime \mu \lambda_{4} \ldots \lambda_{s}} \partial^{\nu} A^{\prime}{ }_{\nu \lambda_{4} \ldots \lambda_{s}}-A_{\lambda_{1} \ldots \lambda_{s}} J^{\lambda_{1} \ldots \lambda_{s}},
\end{aligned}
$$

where $A^{\lambda_{1} \ldots \lambda_{s}}$ is a symmetric Abelian tensor gauge field of rank $s$ and $J^{\lambda_{1} \ldots \lambda_{s}}$ is a symmetric external current. $A^{\prime}$ denotes the trace of the field $A_{\lambda_{3} \ldots \lambda_{s}}^{\prime} \equiv A^{\rho}{ }_{\rho \lambda_{3} \ldots \lambda_{s}}$, while the other notations here should be self-evident. The field is restricted to be double traceless ${ }^{2}$, i.e.

$$
A_{\lambda_{5} \ldots \lambda_{s}}^{\prime \prime} \equiv \eta^{\rho_{1} \rho_{2}} \eta^{\rho_{3} \rho_{4}} A_{\rho_{1} \rho_{2} \rho_{3} \rho_{4} \lambda_{5} \ldots \lambda_{s}}=0 .
$$

The same property is inherited by the current $J^{\lambda_{1} \ldots \lambda_{s}}$, because it is contracted with the field $A_{\lambda_{1} \ldots \lambda_{s}}$ in the action, thus $J_{\lambda_{5} \ldots \lambda_{s}}^{\prime \prime}=0$. These conditions have an effect only for $s \geq 4$.

For $s=0$ the above action corresponds to a massless scalar field interacting with an external current. For $s=1$ only the first two terms contribute and correspond to electrodynamics, and for $s=2$ one obtains linearized gravity

$$
\begin{aligned}
& s=0: \quad S=\int d x^{d} \frac{1}{2} \partial_{\mu} A \partial^{\mu} A-A J, \\
& s=1: \quad S=\int d x^{d} \frac{1}{2} \partial_{\mu} A_{\lambda_{1}} \partial^{\mu} A^{\lambda_{1}}-\frac{1}{2} \partial_{\mu} A^{\mu} \partial^{\nu} A_{\nu}-A_{\mu} J^{\mu},
\end{aligned}
$$

\footnotetext{
${ }^{2}$ It was demonstrated by Fierz and Pauli [5] that in order to have a Lagrangian description of a spin- $s$ boson, one should introduce a traceless rank-s tensor field together with auxiliary traceless fields of all lower ranks. Considering the massless limit of the Singh and Hagen Lagrangian [3] one can prove that the tensors of rank $(s-3)$ and lower decouple and the remaining rank- $s$ and rank- $(s-2)$ tensors can be combined into a single double-traceless field of rank $s$ [2]. Note that there exist unconstrained formulations of the theory, with or without auxiliary fields, which remove the double-traceless constraint [23, 26, 15, 17], but lead to higher derivative or non-local terms.
} 


$$
\begin{aligned}
s=2: \quad S= & \int d x^{d} \frac{1}{2} \partial_{\mu} A_{\lambda_{1} \lambda_{2}} \partial^{\mu} A^{\lambda_{1} \lambda_{2}}-\partial_{\mu} A^{\mu \lambda_{2}} \partial^{\nu} A_{\nu \lambda_{2}} \\
& -A^{\prime} \partial_{\mu} \partial_{\nu} A^{\mu \nu}-\frac{1}{2} \partial_{\mu} A^{\prime} \partial^{\mu} A^{\prime}-A_{\lambda_{1} \lambda_{2}} J^{\lambda_{1} \lambda_{2}} .
\end{aligned}
$$

For $s=3$ it is the Schwinger action and has the following form [1]

$$
\begin{aligned}
s=3: \quad S= & \int d x^{d} \frac{1}{2} \partial_{\mu} A_{\lambda_{1} \lambda_{2} \lambda_{3}} \partial^{\mu} A^{\lambda_{1} \lambda_{2} \lambda_{3}}-\frac{3}{2} \partial_{\mu} A^{\mu \lambda_{2} \lambda_{3}} \partial^{\nu} A_{\nu \lambda_{2} \lambda_{3}} \\
& -3 A_{\lambda_{3} \ldots \lambda_{s}}^{\prime} \partial_{\mu} \partial_{\nu} A^{\mu \nu \lambda_{3} \ldots \lambda_{s}}-\frac{3}{2} \partial_{\mu} A^{\prime}{ }_{\lambda_{3} \ldots \lambda_{s}} \partial^{\mu} A^{\prime \lambda_{3} \ldots \lambda_{s}} \\
& -\frac{3}{4} \partial_{\mu} A^{\prime \mu} \partial^{\nu} A^{\prime}{ }_{\nu}-A_{\lambda_{1} \lambda_{2} \lambda_{3}} J^{\lambda_{1} \lambda_{2} \lambda_{s}} .
\end{aligned}
$$

Finally the Fronsdal action for $s=4$ is

$$
\begin{aligned}
s=4: \quad S= & \int d x^{d} \frac{1}{2} \partial_{\mu} A_{\lambda_{1} \ldots \lambda_{4}} \partial^{\mu} A^{\lambda_{1} \ldots \lambda_{4}}-2 \partial_{\mu} A^{\mu \lambda_{2} \lambda_{3} \lambda_{4}} \partial^{\nu} A_{\nu \lambda_{2} \lambda_{3} \lambda_{4}} \\
& -6 A_{\lambda_{3} \lambda_{4}}^{\prime} \partial_{\mu} \partial_{\nu} A^{\mu \nu \lambda_{3} \lambda_{4}}-3 \partial_{\mu} A^{\prime}{ }_{\lambda_{3} \lambda_{4}} \partial^{\mu} A^{\prime \lambda_{3} \lambda_{4}} \\
& -3 \partial_{\mu} A^{\prime \mu \lambda_{4}} \partial^{\nu} A^{\prime}{ }_{\nu \lambda_{4}}-A_{\lambda_{1} \ldots \lambda_{4}} J^{\lambda_{1} \ldots \lambda_{4}} .
\end{aligned}
$$

As we shall see later, the action (2.1) is gauge invariant with respect to the Abelian gauge transformation

$$
\delta_{\xi} A_{\lambda_{1} \ldots \lambda_{s}} \equiv \sum_{1} \partial_{\lambda_{1}} \xi_{\lambda_{2} \ldots \lambda_{s}}, \quad \xi_{\lambda_{4} \ldots \lambda_{s}}^{\prime}=0
$$

where $\xi_{\lambda_{1} \ldots \lambda_{s-1}}$ is a symmetric gauge parameter of rank $s-1$ and the sum $\sum_{1}$ is over all inequivalent index permutations. The gauge parameter has to be traceless, $\xi^{\prime}=0$, as indicated. With such a restriction on the gauge parameter the class of double-traceless fields $\left\{A: A^{\prime \prime}=0\right\}$ remains intact in the course of gauge transformations. Indeed, the double trace of the field transformation (2.4) is proportional to the trace of the gauge parameter and therefore $\xi^{\prime}$ should vanish. On the other hand, the variation of the action with respect to the transformation (2.4) is also proportional to $\xi^{\prime}$ and vanishes only if $\xi^{\prime}=0$. We shall see this below. Because the gauge parameter $\xi$ is restricted to be traceless, the corresponding symmetry group (2.4) is smaller and as a result the current conservation law is weaker (2.12). It seems that this may endanger the unitarity of the theory and our main concern is to demonstrate, following Schwinger and Fronsdal $[1,2]$, that the theory is nevertheless unitary. Thus even with a smaller symmetry gauge group the theory still stays unitary!

Let us derive the equation of motion. The variation of the action (2.1) reads

$$
\begin{aligned}
\delta S= & \int \delta A^{\lambda_{1} \ldots \lambda_{s}}\left\{-\partial^{2} A_{\lambda_{1} \ldots \lambda_{s}}+s \partial_{\lambda_{1}} \partial^{\nu} A_{\nu \lambda_{2} \ldots \lambda_{s}}-\frac{s(s-1)}{2} \partial_{\lambda_{1}} \partial_{\lambda_{2}} A_{\lambda_{3} \ldots \lambda_{s}}^{\prime}+\right. \\
& \left.-\frac{s(s-1)}{2} \eta_{\lambda_{1} \lambda_{2}}\left(\partial^{\mu} \partial^{\nu} A_{\mu \nu \lambda_{3} \ldots \lambda_{s}}-\partial^{2} A_{\lambda_{3} \ldots \lambda_{s}}^{\prime}-\frac{s-2}{2} \partial_{\lambda_{3}} \partial^{\mu} A_{\mu \lambda_{4} \ldots \lambda_{s}}^{\prime}\right)-J_{\lambda_{1} \ldots \lambda_{s}}\right\} .
\end{aligned}
$$

The variation of $A$ is restricted to be symmetric and double-traceless, therefore the variational derivative $\frac{\delta S}{\delta A}$ is equal to the symmetric and double-traceless part of the terms in the curly bracket. Let us first symmetrize the indices in the curly bracket. This yields

$$
\begin{aligned}
& -\partial^{2} A_{\lambda_{1} \ldots \lambda_{s}}+\sum_{1} \partial_{\lambda_{1}} \partial^{\nu} A_{\nu \lambda_{2} \ldots \lambda_{s}}-\sum_{2} \partial_{\lambda_{1}} \partial_{\lambda_{2}} A_{\lambda_{3} \ldots \lambda_{s}}^{\prime} \\
& \quad-\sum_{2} \eta_{\lambda_{1} \lambda_{2}}\left(\partial^{\mu} \partial^{\nu} A_{\mu \nu \lambda_{3} \ldots \lambda_{s}}-\partial^{2} A_{\lambda_{3} \ldots \lambda_{s}}^{\prime}-\frac{1}{2} \sum_{1} \partial_{\lambda_{3}} \partial^{\mu} A_{\mu \lambda_{4} \ldots \lambda_{s}}^{\prime}\right)=J_{\lambda_{1} \ldots \lambda_{s}} .
\end{aligned}
$$


The symmetrized sums $\sum_{1}$ and $\sum_{2}$ are over all inequivalent index permutations and have $s$ and $s(s-1) / 2$ terms respectively ${ }^{3}$. In order to get the correct equation we have to take also the double-traceless part of the curly bracket. However, it will turn out that the resulting expression (2.6) is already double-traceless. The fact, that the symmetrized terms in the curly bracket in (2.5) are already double-traceless, is a major advantage of this Lagrange formulation. If it were not the case, we would need to project the variation to the double-traceless part ${ }^{4}$. Thus the equation of motion for the Abelian tensor gauge field $A_{\lambda_{1} \ldots \lambda_{s}}$ is indeed the equation (2.6) and it contains a second order linear differential operator $L$ acting on the field $A$

$$
\begin{aligned}
(L A)_{\lambda_{1} \ldots \lambda_{s}} \equiv & -\partial^{2} A_{\lambda_{1} \ldots \lambda_{s}}+\sum_{1} \partial_{\lambda_{1}} \partial^{\nu} A_{\nu \lambda_{2} \ldots \lambda_{s}}-\sum_{2} \partial_{\lambda_{1}} \partial_{\lambda_{2}} A_{\lambda_{3} \ldots \lambda_{s}}^{\prime} \\
& -\sum_{2} \eta_{\lambda_{1} \lambda_{2}}\left(\partial^{\mu} \partial^{\nu} A_{\mu \nu \lambda_{3} \ldots \lambda_{s}}-\partial^{2} A_{\lambda_{3} \ldots \lambda_{s}}^{\prime}-\frac{1}{2} \sum_{1} \partial_{\lambda_{3}} \partial^{\mu} A_{\mu \lambda_{4} \ldots \lambda_{s}}^{\prime}\right),
\end{aligned}
$$

whose double trace is equal to zero $(L A)_{\lambda_{5} \ldots \lambda_{s}}^{\prime \prime} \equiv 0$ (see below in $(2.17)$ ). We can express the equation (2.6) in the operator form as

$$
(L A)_{\lambda_{1} \ldots \lambda_{s}}=J_{\lambda_{1} \ldots \lambda_{s}} .
$$

It follows therefore that the current also should be double traceless

$$
J_{\lambda_{5} \ldots \lambda_{s}}^{\prime \prime}=0 .
$$

This is consistent with the observation made after formula (2.2). These equations completely define the theory and our intention now is to describe the physical properties of the equation (2.6), (2.7).

Let us compute first the divergence of the l.h.s. of the equation (2.6) in order to check if it is equal to zero or not. This will tell us about current divergence $\partial^{\mu} J_{\mu \lambda_{2} \ldots \lambda_{s}}$ through the equation of motion (2.6), (2.7). The straightforward computation gives

$$
\begin{aligned}
-\partial^{\mu} & (L A)_{\mu \lambda_{2} \ldots \lambda_{s}} \\
& =\sum_{2} \eta_{\lambda_{2} \lambda_{3}}\left(\partial^{\mu} \partial^{\nu} \partial^{\rho} A_{\mu \nu \rho \lambda_{4} \ldots \lambda_{s}}-\frac{3}{2} \partial^{\mu} \partial^{2} A_{\mu \lambda_{4} \ldots \lambda_{s}}^{\prime}-\frac{1}{2} \sum_{1} \partial_{\lambda_{4}} \partial^{\mu} \partial^{\nu} A_{\mu \nu \lambda_{4} \ldots \lambda_{s}}^{\prime}\right),
\end{aligned}
$$

and it is obviously not equal to zero. Therefore the current is not conserved in a usual sense: $\partial^{\mu} J_{\mu \lambda_{2} \ldots \lambda_{s}} \neq 0$. The full conservation gets replaced by a weaker condition which becomes transparent after calculating the trace of the divergence:

$$
\begin{aligned}
& -\partial^{\mu}(L A)_{\mu \lambda_{4} \ldots \lambda_{s}}^{\prime} \\
& \quad=(d+2 s-6)\left(\partial^{\mu} \partial^{\nu} \partial^{\rho} A_{\mu \nu \rho \lambda_{4} \ldots \lambda_{s}}-\frac{3}{2} \partial^{\mu} \partial^{2} A_{\mu \lambda_{4} \ldots \lambda_{s}}^{\prime}-\frac{1}{2} \sum_{1} \partial_{\lambda_{4}} \partial^{\mu} \partial^{\nu} A_{\mu \nu \lambda_{4} \ldots \lambda_{s}}^{\prime}\right) .
\end{aligned}
$$

One can clearly see that there is a simple algebraic relation between the divergence (2.9) and trace of the divergence $(2.10)$

$$
\partial^{\mu}(L A)_{\mu \lambda_{2} \ldots \lambda_{s}}-\frac{1}{d+2 s-6} \sum_{2} \eta_{\lambda_{2} \lambda_{3}} \partial^{\mu}(L A)_{\mu \lambda_{4} \ldots \lambda_{s}}^{\prime}=0 .
$$

Because the equation of motion has the form $L A=J$, where $J$ is the current, it follows that the equation is self-consistent and has solutions only if the current obeys the same relation as $L A$, or, in other words, it has to fulfil a weaker current conservation when $s \geq 3[1,2]$

$$
\partial^{\mu} J_{\mu \lambda_{2} \ldots \lambda_{s}}-\frac{1}{d+2 s-6} \sum_{2} \eta_{\lambda_{2} \lambda_{3}} \partial^{\mu} J_{\mu \lambda_{4} \ldots \lambda_{s}}^{\prime}=0 .
$$

\footnotetext{
${ }^{3}$ This is described in more detail in Appendix B.

${ }^{4}$ This projection is given for any rank-s symmetric tensor field in Appendix C.
} 
Thus the current is fully conserved only when $s=1,2$, but for general $s \geq 3$ the current is not conserved in a usual sense because only the traceless part of the current divergence vanishes ${ }^{5}$. Our main concern in the subsequent sections is to demonstrate that this weaker current conservation law guarantees the unitarity of the theory. Physically this means that only waves with transverse polarizations are propagating very far from the sources, as it is the case for fully conserved currents.

It is also true that the equations (2.11) and (2.12) are consequences of the local gauge invariance of the action (2.1) with respect to the above Abelian gauge transformation of the tensor field (2.4). The variation of the kinetic term in the action (2.1) with respect to the transformation $(2.4)$ is

$$
\delta_{\xi} S=\int d^{d} x(L A)_{\lambda_{1} \ldots \lambda_{s}} \delta A^{\lambda_{1} \ldots \lambda_{s}}=-s \int d^{d} x \xi^{\lambda_{2} \ldots \lambda_{s}} \partial^{\lambda_{1}}(L A)_{\lambda_{1} \ldots \lambda_{s}} .
$$

If $\xi$ is traceless, then the contraction with $\xi$ projects to the traceless part of the divergence of $L A$ which as we have seen in (2.11) vanishes, then $\delta_{\xi} S=0$. The gauge invariance of the equation of motion (2.11) and (2.13) and the fact that $L$ is a linear operator implies that any pure gauge field of the form (2.4) is a solution of the homogenous equation $L A=0$. Therefore one can add to any particular solution $A$ of (2.6) a pure gauge field to form a new solution

$$
A_{\lambda_{1} \ldots \lambda_{s}} \rightarrow A_{\lambda_{1} \ldots \lambda_{s}}+\sum_{1} \partial_{\lambda_{1}} \xi_{\lambda_{2} \ldots \lambda_{s}}
$$

Deriving the equation of motion we have used the fact that the expression $L A$ in (2.7) is already double traceless. To check this, notice that the linear operator $L$ can be represented in the form

$$
(L A)_{\lambda_{1} \ldots \lambda_{s}} \equiv\left(L_{0} A\right)_{\lambda_{1} \ldots \lambda_{s}}-\frac{1}{2} \sum_{2} \eta_{\lambda_{1} \lambda_{2}}\left(L_{0} A\right)_{\lambda_{3} \ldots \lambda_{s}}^{\prime}
$$

where $L_{0}$ is given by

$$
\left(L_{0} A\right)_{\lambda_{1} \ldots \lambda_{s}} \equiv-\partial^{2} A_{\lambda_{1} \ldots \lambda_{s}}+\sum_{1} \partial_{\lambda_{1}} \partial^{\nu} A_{\nu \lambda_{2} \ldots \lambda_{s}}-\sum_{2} \partial_{\lambda_{1}} \partial_{\lambda_{2}} A_{\lambda_{3} \ldots \lambda_{s}}^{\prime}
$$

with its trace being

$$
\begin{aligned}
\left(L_{0} A\right)_{\lambda_{3} \ldots \lambda_{s}}^{\prime}= & -2 \partial^{2} A_{\lambda_{3} \ldots \lambda_{s}}^{\prime}+2 \partial^{\mu} \partial^{\nu} A_{\mu \nu \lambda_{3} \ldots \lambda_{s}} \\
& -\sum_{1} \partial_{\lambda_{3}} \partial^{\mu} A_{\mu \lambda_{4} \ldots \lambda_{s}}^{\prime}-\sum_{2} \partial_{\lambda_{1}} \partial_{\lambda_{2}} A_{\lambda_{3} \ldots \lambda_{s}}^{\prime \prime} .
\end{aligned}
$$

The last term vanishes because $A^{\prime \prime}=0$. Calculating the double trace of $\left(L_{0} A\right)$, terms with single traces $A^{\prime}$ of the tensor gauge field all cancel and we get (using the fact that the last term of (2.16) already vanishes)

$$
\left(L_{0} A\right)_{\lambda_{5} \ldots \lambda_{s}}^{\prime \prime}=-2 \partial^{2} A_{\lambda_{5} \ldots \lambda_{s}}^{\prime \prime}-\sum_{1} \partial_{\lambda_{5}} \partial^{\mu} A_{\mu \lambda_{6} \ldots \lambda_{s}}^{\prime \prime}=0 .
$$

\footnotetext{
${ }^{5}$ Remember that $J_{\lambda_{1} \ldots \lambda_{s}}$ is double traceless (2.8). Taking the divergence and taking the trace are commuting operations. Therefore also $\partial^{\mu} J_{\mu \lambda_{2} \ldots \lambda_{s}}$ is double traceless. The traceless part of a double traceless field $A_{\lambda_{1} \ldots \lambda_{s}}$ is given by $A_{\lambda_{1} \ldots \lambda_{s}}-\frac{1}{d+2 s-4} \sum_{2} \eta_{\lambda_{1} \lambda_{2}} A_{\lambda_{3} \ldots \lambda_{s}}^{\prime}$. The divergence of $J$ has only rank $s-1$ which leads to the different prefactor in (2.12). If there were no restriction to double traceless fields, the traceless part would contain subtractions of higher traces as well. The full projection is given in Appendix C.

One should stress that the traceless part of the divergence of the current in (2.12) differs from the divergence of the traceless part $\partial^{\lambda_{1}}\left(J_{\lambda_{1} \ldots \lambda_{s}}-\frac{1}{d+2 s-4} \sum_{2} \eta_{\lambda_{1} \lambda_{2}} J_{\lambda_{3} \ldots \lambda_{s}}^{\prime}\right) \neq 0$, which does not vanish.
} 
Notice that

$$
(L A)_{\lambda_{3} \ldots \lambda_{s}}^{\prime}=-\frac{1}{2}(d+2 s-6)\left(L_{0} A\right)_{\lambda_{3} \ldots \lambda_{s}}^{\prime},
$$

and therefore we have

$$
(L A)_{\lambda_{5} \ldots \lambda_{s}}^{\prime \prime}=-\frac{1}{2}(d+2 s-6)\left(L_{0} A\right)_{\lambda_{5} \ldots \lambda_{s}}^{\prime \prime}=0 .
$$

In summary we have the Lagrangian (2.1), the corresponding equations of motion (2.6) and a weak current conservation (2.12) which is the consequence of the invariance of the action with respect to the Abelian gauge transformations (2.4) with traceless gauge parameters $\xi$.

\section{Solving the equation in de Donder-Fronsdal gauge}

The idea for solving the equation of motion (2.6) in the presence of the external current $J_{\lambda_{1} \ldots \lambda_{s}}$ is to find a possible gauge fixing condition imposed on the field $A_{\lambda_{1} \ldots \lambda_{s}}$ in which the equation of motion reduces to its diagonal form: $-\partial^{2} A_{\lambda_{1} \ldots \lambda_{s}}=(P J)_{\lambda_{1} \ldots \lambda_{s}}$. In order to realize this program one should make two important steps [2]. The first step is to represent the linear differential operator $L$ in (2.14) as a product of two operators $R$ and $L_{0}$

$$
\left(R L_{0} A\right)_{\lambda_{1} \ldots \lambda_{s}}=J_{\lambda_{1} \ldots \lambda_{s}}
$$

where the operator $R$

$$
(R A)_{\lambda_{1} \ldots \lambda_{s}} \equiv A_{\lambda_{1} \ldots \lambda_{s}}-\frac{1}{2} \sum_{2} \eta_{\lambda_{1} \lambda_{2}} A_{\lambda_{3} \ldots \lambda_{s}}^{\prime}
$$

is a nonsingular algebraic operator with its inverse $P$

$$
(P A)_{\lambda_{1} \ldots \lambda_{s}} \equiv A_{\lambda_{1} \ldots \lambda_{s}}-\frac{1}{(d+2 s-6)} \sum_{2} \eta_{\lambda_{1} \lambda_{2}} A_{\lambda_{3} \ldots \lambda_{s}}^{\prime}
$$

and $L_{0}$ is the second order differential operator given in (2.15). The second step is to represent the operator $L_{0}$ in the following form

$$
\left(L_{0} A\right)_{\lambda_{1} \ldots \lambda_{s}}=-\partial^{2} A_{\lambda_{1} \ldots \lambda_{s}}+\sum_{1} \partial_{\lambda_{1}}\left(\partial^{\nu} A_{\nu \lambda_{2} \ldots \lambda_{s}}-\frac{1}{2} \sum_{1} \partial_{\lambda_{2}} A_{\lambda_{3} \ldots \lambda_{s}}^{\prime}\right) .
$$

From the last expression one can deduce that if we could impose the gauge condition on the gauge field $A$ of the form

$$
\partial^{\mu} A_{\mu \lambda_{2} \ldots \lambda_{s}}-\frac{1}{2} \sum_{1} \partial_{\lambda_{2}} A_{\lambda_{3} \ldots \lambda_{s}}^{\prime}=0
$$

then the operator $L_{0}$ would reduce to the d'Alembertian:

$$
\left(L_{0} A\right)_{\lambda_{1} \ldots \lambda_{s}}=-\partial^{2} A_{\lambda_{1} \ldots \lambda_{s}},
$$

and the equation of motion $R L_{0} A=R\left(-\partial^{2}\right) A=J$ can be solved by using the inverse operator $P$. Thus we have

$$
-\partial^{2} A_{\lambda_{1} \ldots \lambda_{s}}=(P J)_{\lambda_{1} \ldots \lambda_{s}} .
$$

In momentum space, where $-\partial^{2} \rightarrow k^{2}$, a solution to the above equation is given by the formula

$$
A_{\lambda_{1} \ldots \lambda_{s}}=\frac{(P J)_{\lambda_{1} \ldots \lambda_{s}}}{k^{2}}
$$


The crucial question about the gauge fixing condition (3.3) is, whether it is accessible or not. Let us see how that expression transforms under the gauge transformation (3.3)

$$
\delta_{\xi}\left(\partial^{\mu} A_{\mu \lambda_{2} \ldots \lambda_{s}}-\frac{1}{2} \sum_{1} \partial_{\lambda_{2}} A_{\lambda_{3} \ldots \lambda_{s}}^{\prime}\right)=\square \xi_{\lambda_{2} \ldots \lambda_{s}} .
$$

It is obvious that, if the l.h.s. is not equal to zero, then one can always find a solution $\xi$ so as to fulfil the gauge condition (3.3). Let us call it de Donder-Fronsdal gauge, because for $s=2$ it coincides with de Donder gauge in gravity ${ }^{6}$.

\section{Interaction of higher spin field with external currents}

With the solution (3.4) at hand we can find out the properties of the field $A$ propagating far from the current $J$ when the latter is constrained to be weakly conserved (2.12). The main result of $[1,2]$ is that only transverse degrees of freedom propagate to infinity, even when the current is only weakly conserved (2.12). For completeness let us recollect the corresponding results for the lower-rank gauge fields $[4,1]$ and then present the proof of [2] for the general case.

In electrodynamics $(s=1)$ and linearized gravity $(s=2)(2.3)$ the currents are fully conserved

$$
k^{\mu} J_{\mu}=0, \quad k^{\mu} J_{\mu \nu}=0
$$

and the interaction between currents can be straightforwardly analyzed [4]. But already for the Schwinger equation of rank-3 gauge fields the weaker conservation (2.12) takes place [1]

$$
k^{\mu} J_{\mu \nu \lambda}-\frac{1}{d} \eta_{\nu \lambda} k^{\mu} J_{\mu}^{\prime}=0
$$

Thus we have to consider two cases: when the currents are fully conserved (4.1) and the case when it is weakly conserved (4.2).

In the general action (2.1) the interaction term of the gauge field with the current $\tilde{J}$ is of the form $-A \tilde{J}$, therefore the exchange interaction between two currents $J$ and $\tilde{J}$ can be found with the help of the gauge field generated by a source $J$ in (3.4)

$$
-A_{\rho_{1} \ldots \rho_{s}} \tilde{J}^{\rho_{1} \ldots \rho_{s}}=-J^{\lambda_{1} \ldots \lambda_{s}} \frac{P_{\lambda_{1} \ldots \lambda_{s}, \rho_{1} \ldots \rho_{s}}}{k^{2}} \tilde{J}^{\rho_{1} \ldots \rho_{s}}
$$

with the expression

$$
\Delta_{\lambda_{1} \ldots \lambda_{s}, \rho_{1} \ldots \rho_{s}}(k)=\frac{P_{\lambda_{1} \ldots \lambda_{s}, \rho_{1} \ldots \rho_{s}}}{k^{2}}
$$

representing the propagator of the rank- $s$ gauge field. The symmetric operator $P$ is given by (3.2). For the lower-rank fields the interaction has the following form

$$
\begin{aligned}
& s=1 \quad-J^{\lambda} \frac{\eta_{\lambda \rho}}{k^{2}} \tilde{J}^{\rho}, \\
& s=2 \quad-J^{\lambda_{1} \lambda_{2}} \frac{\eta_{\lambda_{1} \rho_{1}} \eta_{\lambda_{2} \rho_{2}}-\frac{1}{d-2} \eta_{\lambda_{1} \lambda_{2}} \eta_{\rho_{1} \rho_{2}}}{k^{2}} \tilde{J}^{\rho_{1} \rho_{2}},
\end{aligned}
$$

\footnotetext{
${ }^{6}$ In contrast to the gravity case $s=2$, the gauge fixing condition (3.3) for general $s$ cannot be written as the divergence of $A_{\lambda_{1} \ldots \lambda_{s}}-\frac{1}{2} \sum_{2} \eta_{\lambda_{1} \lambda_{2}} A_{\lambda_{3} \ldots \lambda_{s}}^{\prime}$. However, it can be written as the traceless part of its divergence$$
\Pi_{\rho_{2} \ldots \rho_{s}}{ }^{\lambda_{2} \ldots \lambda_{s}} \partial^{\lambda_{1}}\left(A_{\lambda_{1} \ldots \lambda_{s}}-\frac{1}{2} \sum_{2} \eta_{\lambda_{1} \lambda_{2}} A_{\lambda_{3} \ldots \lambda_{s}}^{\prime}\right)=0
$$

Here $\Pi$ is the projector to the traceless part given in Appendix C. 


$$
s=3-J^{\lambda_{1} \lambda_{2} \lambda_{3}} \frac{\eta_{\lambda_{1} \rho_{1}} \eta_{\lambda_{2} \rho_{2}} \eta_{\lambda_{3} \rho_{3}}-\frac{3}{d} \eta_{\lambda_{1} \rho_{1}} \eta_{\lambda_{2} \lambda_{3}} \eta_{\rho_{2} \rho_{3}}}{k^{2}} \tilde{J}^{\rho_{1} \rho_{2} \rho_{3}}
$$

To simplify the analysis of this interaction we can always take the momentum vector $k$ in the form:

$$
k_{\mu}=(\omega, 0, \ldots, 0, \kappa)
$$

and introduce the parity reversed momentum vector

$$
\bar{k}_{\mu}=(\omega, 0, \ldots, 0,-\kappa)
$$

together with $d-2$ space-like orthogonal vectors $e_{i}^{\mu}, i=1, \ldots, d-2$ :

$$
\begin{aligned}
& e_{1}^{\mu}=(0,1, \ldots 0,0), \\
& \cdots \ldots \ldots \ldots \ldots \ldots \\
& e_{d-2}^{\mu}=(0,0, \ldots, 1,0) .
\end{aligned}
$$

These vectors form a frame and the metric tensor can be represented in the form

$$
\eta^{\mu \nu}=-\sum_{i} e_{i}^{\mu} e_{i}^{\nu}+\frac{(k+\bar{k})^{\mu}(k+\bar{k})^{\nu}}{2\left(k^{2}+k \bar{k}\right)}+\frac{(k-\bar{k})^{\mu}(k-\bar{k})^{\nu}}{2\left(k^{2}-k \bar{k}\right)},
$$

where the first term projects to the transversal plane, while the remaining ones project to the longitudinal direction. On the mass-shell $k^{2}=\bar{k}^{2}=\omega^{2}-\kappa^{2}=0$ this expression reduces to the familiar expression [1]

$$
\eta^{\mu \nu}=-\sum_{i} e_{i}^{\mu} e_{i}^{\nu}+\frac{k^{\mu} \bar{k}^{\nu}+\bar{k}^{\mu} k^{\nu}}{k \bar{k}} .
$$

Armed with the last two expressions one can prove that only transversal polarizations of the tensor gauge boson participate in the exchange interaction between currents at large distances, when $k^{2} \approx 0$. Indeed, inserting the representation (4.8) into (4.4) and (4.5) and using the current conservation, which is valid in these cases (4.1), we shall get

$$
\begin{aligned}
s=1 & -J^{\lambda} \frac{\eta_{\lambda \rho}}{k^{2}} \tilde{J}^{\rho}=\frac{J_{\lambda} e_{i}^{\lambda} e_{i}^{\rho} \tilde{J}_{\rho}}{\omega^{2}-\kappa^{2}}=\frac{J_{i} \tilde{J}_{i}}{\omega^{2}-\kappa^{2}}, \\
s=2 \quad-J^{\lambda_{1} \lambda_{2}} \frac{\eta_{\lambda_{1} \rho_{1}} \eta_{\lambda_{2} \rho_{2}}-\frac{1}{d-2} \eta_{\lambda_{1} \lambda_{2}} \eta_{\rho_{1} \rho_{2}}}{k^{2}} \tilde{J}^{\rho_{1} \rho_{2}} & \\
& =-\frac{J_{\lambda_{1} \lambda_{2}} e_{i}^{\lambda_{1}} e_{j}^{\lambda_{2}} e_{i}^{\rho_{1}} e_{j}^{\rho_{2}} \tilde{J}_{\rho_{1} \rho_{2}}-\frac{1}{d-2} J_{\lambda_{1} \lambda_{2}} e_{i}^{\lambda_{1}} e_{i}^{\lambda_{2}} e_{j}^{\rho_{1}} e_{j}^{\rho_{2}} \tilde{J}_{\rho_{1} \rho_{2}}}{\omega^{2}-\kappa^{2}}=-\frac{J_{i j} \tilde{J}_{i j}-\frac{1}{d-2} J_{i i} \tilde{J}_{j j}}{\omega^{2}-\kappa^{2}} .
\end{aligned}
$$

All bilinear terms $k_{\mu} \bar{k}_{\nu}$ are cancelled because of the current conservation, and quantities

$$
J_{i}=J_{\lambda} e_{i}^{\lambda}, \quad J_{i j}=J_{\lambda_{1} \lambda_{2}} e_{i}^{\lambda_{1}} e_{j}^{\lambda_{2}}
$$

are projection of currents to the transverse plane. At the pole $\omega^{2}-\kappa^{2}=0$ the residues are positive definite. Indeed, for $s=1$ we have $J_{i} J_{i}$ and for $s=2$ the numerator can be written as a square of the traceless part of $J_{i j}$

$$
\left(J_{i j}-\frac{1}{d-2} \delta_{i j} J_{n n}\right)\left(J_{i j}-\frac{1}{d-2} \delta_{i j} J_{m m}\right) .
$$

It is obvious, how to extended this proof to the higher-rank fields, if the corresponding currents would be fully conserved, but unfortunately they are not! What is amazing nevertheless, is 
that for weakly conserved currents $(2.12)$, (4.2) the analysis can be reduced to the case of fully conserved currents. Therefore it is worth to follow the general Schwinger consideration of the exchange interaction between conserved currents [1]. The general form of the exchange interaction (4.3) is

$$
\begin{aligned}
-J^{\lambda_{1} \ldots \lambda_{s}} & \frac{P_{\lambda_{1} \ldots \lambda_{s}, \rho_{1} \ldots \rho_{s}}}{k^{2}} \tilde{J}^{\rho_{1} \ldots \rho_{s}} \\
& =-J^{\lambda_{1} \ldots \lambda_{s}} \frac{\left(\eta_{\lambda_{1} \rho_{1}} \ldots \eta_{\lambda_{s} \rho_{s}}-\frac{s(s-1)}{2(d+2 s-6)} \eta_{\lambda_{1} \lambda_{2}} \eta_{\rho_{1} \rho_{2}} \eta_{\lambda_{3} \rho_{3}} \ldots \eta_{\lambda_{s} \rho_{s}}\right)}{k^{2}} \tilde{J}^{\rho_{1} \ldots \rho_{s}},
\end{aligned}
$$

where we have used the expression for the matrix $P$ in (3.2). Again inserting the representation (4.8) for the metric tensor into the (4.9) and supposing that the currents are conserved: $k^{\mu} J_{\mu \lambda_{2} \ldots \lambda_{s}}=0, k^{\mu} \tilde{J}_{\mu \lambda_{2} \ldots \lambda_{s}}=0$, we shall get

$$
(-)^{s+1} \frac{J_{i_{1} \ldots i_{s}} \tilde{J}_{i_{1} \ldots i_{s}}-\frac{s(s-1)}{2(d+2 s-6)} J_{i_{3} \ldots i_{s}}^{\prime} \tilde{J}_{i_{3} \ldots i_{s}}^{\prime}}{\omega^{2}-\kappa^{2}},
$$

where

$$
J_{i_{1} \ldots i_{s}}=J_{\lambda_{1} \ldots \lambda_{s}} e_{i_{1}}^{\lambda_{1}} \cdots e_{i_{s}}^{\lambda_{s}} .
$$

The longitudinal modes $k_{\mu} \bar{k}_{\nu}$ do not contribute because of the current conservation and we are left with only transversal propagating modes! The expression in the above equation coincides with the product of the traceless parts of the currents, as it was for $s=2$. Indeed, the trace has reduced to the transversal directions, and the effective dimension has therefore reduced by 2, and the coefficient $\frac{s(s-1)}{2(d+2 s-6)}$ is the correct coefficient for the traceless projector for fields of rank $s$ in dimension $d-2 .^{7}$ Our main concern in the next section is to prove that almost the same mechanism works in the case of the weakly conserved currents $[1,2]$.

In the above discussion we have considered interactions at large distances, when $k^{2}=$ $\omega^{2}-\kappa^{2} \approx 0$, therefore keeping the most singular terms. In order to analyze the short distance behaviour, when $\omega^{2}-k^{2} \neq 0$, one should use the relation (4.7) and follow the beautiful consideration of Feynman [4].

\section{Interaction of weakly conserved currents}

In order to prove that in the case of weakly conserved currents the propagating modes are positive definite transversal polarizations we have to reformulate the exchange interaction (4.3)

$$
-J^{\lambda_{1} \ldots \lambda_{s}} \frac{P_{\lambda_{1} \ldots \lambda_{s}, \rho_{1} \ldots \rho_{s}}}{k^{2}} \tilde{J}^{\rho_{1} \ldots \rho_{s}}
$$

in a way that it becomes [2]

$$
-J_{f} \lambda_{1} \ldots \lambda_{s} \frac{P_{\lambda_{1} \ldots \lambda_{s}, \rho_{1} \ldots \rho_{s}}}{k^{2}} \tilde{J}_{f}^{\rho_{1} \ldots \rho_{s}}
$$

where the effective current $J_{f}$ is fully conserved. Let us introduce the projection $\Pi$ to the traceless part, which we already used implicitly several times. Its action on double traceless tensor $t_{\lambda_{1} \ldots \lambda_{s-1}}$ of rank $s-1$ is given by ${ }^{8}$

$$
\Pi_{\lambda_{1} \ldots \lambda_{s-1}}^{\rho_{1} \ldots \rho_{s-1}} t_{\rho_{1} \ldots \rho_{s-1}}=t_{\rho_{1} \ldots \rho_{s-1}}-\frac{1}{d+2 s-6} \sum_{2} \eta_{\rho_{1} \rho_{2}} t_{\rho_{3} \ldots \rho_{s-1}}^{\prime}
$$

\footnotetext{
${ }^{7}$ Compare with Appendix C.

${ }^{8}$ As described in Appendix C, higher traces appear in the projection, if $A$ is not double traceless.
} 
and we can represent the weak current conservation (2.12) in the following form

$$
\Pi^{\lambda_{2} \ldots \lambda_{s}}{ }_{\rho_{2} \ldots \rho_{s}} k_{\mu} J^{\mu \rho_{2} \ldots \rho_{s}}=0,
$$

with $t_{\lambda_{2} \ldots \lambda_{s}}=k_{\mu} J^{\mu \rho_{2} \ldots \rho_{s}}$. This equation can be contracted with an arbitrary tensor $f_{\lambda_{2} \ldots \lambda_{s}}$ of rank $s-1$, and because $\Pi$ is a symmetric matrix this can be written as

$$
k_{\rho_{1}}(\Pi f)_{\rho_{2} \ldots \rho_{s}} J^{\rho_{1} \ldots \rho_{s}}=0,
$$

that is, the contraction of $\frac{1}{s} \sum_{1} k_{\rho_{1}}(\Pi f)_{\rho_{2} \ldots \rho_{s}}$ with the current vanishes for all $f$. The interpretation of this formula is, that instead of the longitudinal operator $k_{\rho_{1}}$ in the case of fully conserved currents, we have the operator $\frac{1}{s} \sum_{1} k_{\rho_{1}}(\Pi f)_{\rho_{2} \ldots \rho_{s}}$ which plays a similar role.

Now one can add this operator to the current $J$ to form an effective current $J_{f}$

$$
J_{f}^{\lambda_{1} \ldots \lambda_{s}}=J^{\lambda_{1} \ldots \lambda_{s}}+R^{\lambda_{1} \ldots \lambda_{s}, \rho_{1} \ldots \rho_{s}} k_{\rho_{1}}(\Pi f)_{\rho_{2} \ldots \rho_{s}}
$$

where $R$ was defined in (3.1). The interaction of the effective currents $J_{f} P \tilde{J}_{f}$ will be identical with the original interaction of currents $J P \tilde{J}$, if the cross terms and the square of the additional operator vanish. The cross terms will vanish, because they simply express the weak current conservation (5.1). For the square we have

$$
\begin{aligned}
& k^{\rho_{1}}(\Pi f)^{\rho_{2} \ldots \rho_{s}} R_{\rho_{1} \ldots \rho_{s}, \lambda_{1} \ldots \lambda_{s}} k^{\lambda_{1}}(\Pi \tilde{f})^{\lambda_{2} \ldots \lambda_{s}} \\
& =\frac{1}{s} \sum_{1} k^{\rho_{1}}(\Pi f)^{\rho_{2} \ldots \rho_{s}}\left(\eta_{\rho_{1} \lambda_{1}} \cdots \eta_{\rho_{s} \lambda_{s}}-\frac{s(s-1)}{4} \eta_{\rho_{1} \rho_{2}} \eta_{\lambda_{1} \lambda_{2}} \eta_{\rho_{3} \lambda_{3}} \cdots \eta_{\rho_{s} \lambda_{s}}\right) \frac{1}{s} \sum_{1} k^{\lambda_{1}}(\Pi \tilde{f})^{\lambda_{2} \ldots \lambda_{s}} \\
& =\frac{1}{s} k^{2}(\Pi f)_{\lambda_{1} \ldots \lambda_{s}}(\Pi \tilde{f})^{\lambda_{1} \ldots \lambda_{s}}
\end{aligned}
$$

where we have used the fact that $(\Pi f)$ is traceless. It vanishes on the mass-shell $k^{2}=0$. Therefore we have

$$
\begin{aligned}
-J_{f} & \lambda_{1} \ldots \lambda_{s} \frac{P_{\lambda_{1} \ldots \lambda_{s}, \rho_{1} \ldots \rho_{s}}}{k^{2}} \tilde{J}_{f}^{\rho_{1} \ldots \rho_{s}}=-(J+k(\Pi f) R)^{\lambda_{1} \ldots \lambda_{s}} \frac{P_{\lambda_{1} \ldots \lambda_{s}, \rho_{1} \ldots \rho_{s}}}{k^{2}}(\tilde{J}+R k(\Pi \tilde{f}))^{\rho_{1} \ldots \rho_{s}} \\
& =-J^{\lambda_{1} \ldots \lambda_{s}} \frac{P_{\lambda_{1} \ldots \lambda_{s}, \rho_{1} \ldots \rho_{s}}}{k^{2}} \tilde{J}^{\rho_{1} \ldots \rho_{s}}-\frac{k_{\rho_{1}}(\Pi f)_{\rho_{2} \ldots \rho_{s}} \tilde{J}^{\rho_{1} \ldots \rho_{s}}}{k^{2}}-\frac{J_{\rho_{1} \ldots \rho_{s}} k^{\rho_{1}}(\Pi \tilde{f})^{\rho_{2} \ldots \rho_{s}}}{k^{2}} \\
& -k^{\rho_{1}}(\Pi f)^{\rho_{2} \ldots \rho_{s}} \frac{R_{\rho_{1} \ldots \rho_{s}, \lambda_{1} \ldots \lambda_{s}}}{k^{2}} k^{\lambda_{1}}(\Pi \tilde{f})^{\lambda_{2} \ldots \lambda_{s}} .
\end{aligned}
$$

The last three terms are equal to zero, as we already explained, and the equivalence of the interaction has been demonstrated with the effective current (5.2). Let us calculate now the divergence of the effective current

$$
\begin{aligned}
k_{\lambda_{1}} J_{f}^{\lambda_{1} \ldots \lambda_{s}} & =k_{\lambda_{1}} J^{\lambda_{1} \ldots \lambda_{s}}+k_{\lambda_{1}} R^{\lambda_{1} \ldots \lambda_{s}, \rho_{1} \ldots \rho_{s}} k_{\rho_{1}}(\Pi f)_{\rho_{2} \ldots \rho_{s}} \\
& =\frac{1}{d+2 s-6} \sum_{2} \eta^{\lambda_{2} \lambda_{3}} k_{\mu} J^{\prime \mu \lambda_{4} \ldots \lambda_{s}}+\frac{1}{s} k^{2}(\Pi f)^{\lambda_{2} \ldots \lambda_{s}}-\frac{1}{s} \sum_{2} \eta^{\lambda_{2} \lambda_{3}} k_{\mu} k_{\nu}(\Pi f)^{\mu \nu \lambda_{4} \ldots \lambda_{s}} \\
& =\frac{1}{d+2 s-6} \sum_{2} \eta^{\lambda_{2} \lambda_{3}} k_{\mu} J^{\prime \mu \lambda_{4} \ldots \lambda_{s}}-\frac{1}{s} \sum_{2} \eta^{\lambda_{2} \lambda_{3}} k_{\mu} k_{\nu}(\Pi f)^{\mu \nu \lambda_{4} \ldots \lambda_{s}} .
\end{aligned}
$$

Choosing a tensor $f_{\lambda_{2} \ldots \lambda_{s}}$ so that ${ }^{9}$

$$
\frac{1}{s} k_{\nu}(\Pi f)^{\nu \mu \lambda_{4} \ldots \lambda_{s}}=\frac{1}{(d+2 s-6)} J^{\prime} \mu \lambda_{4} \ldots \lambda_{s},
$$

we can get a conserved (on mass-shell) effective current

$$
k_{\lambda_{1}} J_{f}^{\lambda_{1} \ldots \lambda_{s}}=0
$$

\footnotetext{
${ }^{9}$ We will provide an explicit solution of this equation in Appendix A.
} 
Thus the interaction $J P \tilde{J}$ can be reduced to the form $J_{f} P \tilde{J}_{f}$, where $J_{f}$ is a conserved current and the problem reduces to the one that we already solved in the previous section ${ }^{10}$.

Summarizing we have to stress that the self-consistency of the equations of motion (2.6), (2.7) of this theory requires the existence of a double-traceless (2.8) and weakly conserved current (2.12). It is outside of the scope of this theory to answer the question whether such external currents exist. It should be answered by a fully interacting theory, a subject of current research in higher spin field theory. In this context we have to remark also that the above consideration does not contradict the Weinberg argument on the non-existence of a fully conserved higher rank current of a specific form [32, p. 538], [31].

\section{Alternative representation of Schwinger-Fronsdal action}

The action (2.1) is a generalization of ordinary Abelian gauge theory and it is therefore tempting to try to write it as a square of some field strength tensor. Field strength tensors are characterized by the property that they transform homogenously under gauge transformations. This means for Abelian gauge transformations that they should not transform at all. Indeed such objects can be constructed, but unfortunately they need to be of $s$-th derivative order [14]. This is like in gravity, where the curvature is of second derivative order of the metric. Square of such objects for $s>1$ certainly cannot coincide with the second order action (2.1). Nevertheless they can be used to construct a nonlocal geometric action which is related to Fronsdal's upon partial gauge fixing $[26,23]$.

We shall try to write Schwinger-Fronsdal's Lagrangian as a square, or at least as a sum of squares, of objects that reduce for $s=1$ to the ordinary field strength tensor. To this end, let us define

$$
\begin{aligned}
& F_{\mu \nu, \lambda_{2} \ldots \lambda_{s}} \equiv \partial_{\mu} A_{\nu \lambda_{2} \ldots \lambda_{s}}-\partial_{\nu} A_{\mu \lambda_{2} \ldots \lambda_{s}}, \\
& F_{\mu \nu, \lambda_{4} \ldots \lambda_{s}}^{\prime} \equiv \partial_{\mu} A_{\nu \lambda_{4} \ldots \lambda_{s}}^{\prime}-\partial_{\nu} A_{\mu \lambda_{4} \ldots \lambda_{s}}^{\prime}, \\
& H_{\mu, \lambda_{3} \ldots \lambda_{s}} \equiv \partial^{\nu} A_{\nu \mu \lambda_{3} \ldots \lambda_{s}}-\frac{s}{2} \partial_{\mu} A_{\lambda_{3} \ldots \lambda_{s}}^{\prime}
\end{aligned}
$$

Their squares read

$$
\begin{aligned}
F_{\mu \nu, \lambda_{2} \ldots \lambda_{s}} F^{\mu \nu, \lambda_{2} \ldots \lambda_{s}} & =\left(\partial_{\mu} A_{\nu \lambda_{2} \ldots \lambda_{s}}-\partial_{\nu} A_{\mu \lambda_{2} \ldots \lambda_{s}}\right)\left(\partial^{\mu} A^{\nu \lambda_{2} \ldots \lambda_{s}}-\partial^{\nu} A^{\mu \lambda_{2} \ldots \lambda_{s}}\right) \\
& =2 \partial_{\mu} A_{\nu \lambda_{2} \ldots \lambda_{s}} \partial^{\mu} A^{\nu \lambda_{2} \ldots \lambda_{s}}-2 \partial_{\mu} A_{\nu \lambda_{2} \ldots \lambda_{s}} \partial^{\nu} A^{\mu \lambda_{2} \ldots \lambda_{s}}, \\
F_{\mu \nu, \lambda_{4} \ldots \lambda_{s}}^{\prime} F^{\mu \nu, \lambda_{4} \ldots \lambda_{s}} & =\left(\partial_{\mu} A_{\nu \lambda_{4} \ldots \lambda_{s}}^{\prime}-\partial_{\nu} A_{\mu \lambda_{4} \ldots \lambda_{s}}^{\prime}\right)\left(\partial^{\mu} A^{\prime \nu \lambda_{4} \ldots \lambda_{s}}-\partial^{\nu} A^{\prime \mu \lambda_{4} \ldots \lambda_{s}}\right) \\
& =2 \partial_{\mu} A_{\nu \lambda_{4} \ldots \lambda_{s}}^{\prime} \partial^{\mu} A^{\prime \nu \lambda_{4} \ldots \lambda_{s}}-2 \partial_{\mu} A_{\nu \lambda_{4} \ldots \lambda_{s}}^{\prime} \partial^{\nu} A^{\prime \mu \lambda_{4} \ldots \lambda_{s}}, \\
H_{\mu, \lambda_{3} \ldots \lambda_{s}} H^{\mu, \lambda_{3} \ldots \lambda_{s}}= & \left(\partial^{\nu} A_{\nu \mu \lambda_{3} \ldots \lambda_{s}}-\frac{s}{2} \partial_{\mu} A_{\lambda_{3} \ldots \lambda_{s}}^{\prime}\right)\left(\partial_{\rho} A^{\rho \mu \lambda_{3} \ldots \lambda_{s}}-\frac{s}{2} \partial^{\mu} A^{\prime \lambda_{3} \ldots \lambda_{s}}\right) \\
= & \partial^{\nu} A_{\nu \mu \lambda_{3} \ldots \lambda_{s}} \partial_{\rho} A^{\rho \mu \lambda_{3} \ldots \lambda_{s}}-s \partial^{\nu} A_{\nu \mu \lambda_{3} \ldots \lambda_{s}} \partial^{\mu} A^{\prime \lambda_{3} \ldots \lambda_{s}} \\
& +\left(\frac{s}{2}\right)^{2} \partial_{\mu} A_{\lambda_{3} \ldots \lambda_{s}}^{\prime} \partial^{\mu} A^{\prime \lambda_{3} \ldots \lambda_{s}},
\end{aligned}
$$

and the Schwinger-Fronsdal action (2.1) can therefore be written as

$$
S=\int d x^{d} \frac{1}{4} F^{2}-\frac{s-1}{2} H^{2}+\frac{s(s-1)(s-2)}{16} F^{2} .
$$

Despite the fact that these field strength tensors do not transform homogeneously, the sum does. Similar field strength tensors have been introduced in [5] and recently in [27, 28, 29, 30].

\footnotetext{
${ }^{10} \mathrm{~A}$ discussion of current interaction in the unconstrained formulation can be found in [24].
} 


\section{A Accessibility of a conserved effective current}

An essential ingredient of the construction of a conserved effective current in Section 5 was the claim in (5.3) that one can choose a tensor $f$ such that

$$
k_{\mu}(\Pi f)^{\mu \lambda_{3} \ldots \lambda_{s}}=\frac{s}{(d+2 s-6)} J^{\prime \lambda_{3} \ldots \lambda_{s}} .
$$

The solution can be derived by expanding the tensors $f$ and $J^{\prime}$ in the basis $e_{i}^{\mu}, k^{\mu}$ and $\bar{k}^{\mu}$ and then compare the coefficients on both sides. For $s=3$ where $f$ has rank 2 and $J^{\prime}$ has rank 1 this yields the following result

$$
\frac{d}{3} f^{\mu \nu}=\frac{2 \bar{k}_{\rho} J^{\prime \rho}}{(k \bar{k})^{2}}\left(k^{\mu} \bar{k}^{\nu}+\bar{k}^{\mu} k^{\nu}\right)+\frac{k_{\rho} J^{\prime \rho}}{(k \bar{k})^{2}} \bar{k}^{\mu} \bar{k}^{\nu}+\sum_{i} \frac{J_{i}^{\prime}}{k \bar{k}}\left(\bar{k}^{\mu} e_{i}^{\nu}+e_{i}^{\mu} \bar{k}^{\nu}\right)-\eta^{\mu \nu} \frac{\bar{k}_{\rho} J^{\prime \rho}}{k \bar{k}} .
$$

In order to avoid too many prefactors in the following, let us introduce the symbol $X$ for the tensor on the righthand side of (A.1)

$$
\frac{s}{d+2 s-6} J^{\prime \lambda_{1} \ldots \lambda_{s-2}} \equiv X^{\lambda_{1} \ldots \lambda_{s-2}} .
$$

For general $s$, the expansion of $f$ and $X$ in the basis takes the form

$$
\begin{aligned}
f_{\lambda_{1} \ldots \lambda_{s-1}}= & \sum_{n_{k}=0}^{s-1} \sum_{n_{\bar{k}}=0}^{s-1-n_{k}} f_{\left(n_{k}\right)\left(n_{\bar{k}}\right) i_{1} \ldots i_{s-1-n_{k}-n_{\bar{k}}}} \\
& \times \sum_{\left(n_{k}\right)}\left(k_{\lambda_{1}} \cdots k_{\lambda_{n_{k}}}\right) \sum_{\left(n_{\bar{k}}\right)}\left(\bar{k}_{\lambda_{n_{k}+1}} \cdots \bar{k}_{\lambda_{n_{k}+n_{\bar{k}}}}\right) e_{\lambda_{n_{k}+n_{\bar{k}}+1}}^{i_{1}} \cdots e_{\lambda_{s-1}}^{i_{s-1-n_{k}-n_{\bar{k}}}} \\
X_{\lambda_{1} \ldots \lambda_{s-2}}= & \sum_{n_{k}=0}^{s-2} \sum_{n_{\bar{k}}=0}^{s-2-n_{k}} X_{\left(n_{k}\right)\left(n_{\bar{k}}\right) i_{1} \ldots i_{s-2-n_{k}-n_{\bar{k}}}} \\
& \times \sum_{\left(n_{k}\right)}\left(k_{\lambda_{1}} \cdots k_{\lambda_{n_{k}}}\right) \sum_{\left(n_{\bar{k}}\right)}\left(\bar{k}_{\lambda_{n_{k}+1}} \cdots \bar{k}_{\lambda_{n_{k}+n_{\bar{k}}}}\right) e_{\lambda_{n_{k}+n_{\bar{k}}+1}^{i_{1}} \cdots e_{\lambda_{s-2}}^{i_{s-2-n_{k}-n_{\bar{k}}}}}
\end{aligned}
$$

The symmetrized sums $\sum_{(n)}$ are over all inequivalent index permutations and are discussed in Appendix B. Let us first find the general solution for the equation for unrestricted $f$

$$
k_{\mu} f^{\mu \lambda_{2} \ldots \lambda_{s-1}}=X^{\lambda_{2} \ldots \lambda_{s-1}}
$$

and put the traceless condition only in the end. Plugging the expansions (A.3) and (A.4) into the above equation leads to the conditions

$$
f_{\left(n_{k}\right)\left(n_{\bar{k}}\right) i_{1} \ldots i_{s-1-n_{k}-n_{\bar{k}}}}=\frac{1}{k \bar{k}} X_{\left(n_{k}\right)\left(n_{\bar{k}}-1\right) i_{1} \ldots i_{s-1-n_{k}-n_{\bar{k}}}} \quad \forall n_{\bar{k}} \geq 1 .
$$

The expansion coefficients $f_{\left(n_{k}\right)(0) i_{1} \ldots i_{s-1-n_{k}}}$ remain undetermined and can be used to make the solution traceless, as we will see now. The trace of $f$ in (A.3) is given by

$$
\begin{aligned}
f_{\lambda_{1} \ldots \lambda_{s-3}}^{\prime}= & \sum_{n_{k}=0}^{s-3} \sum_{n_{\bar{k}}=0}^{s-3-n_{k}}\left(2(k \bar{k}) f_{\left(n_{k}+1\right)\left(n_{\bar{k}}+1\right) i_{1} \ldots i_{s-3-n_{k}-n_{\bar{k}}}}-\sum_{j=1}^{d-2} f_{\left(n_{k}\right)\left(n_{\bar{k}}\right) i_{1} \ldots i_{s-3-n_{k}-n_{\bar{k}}} j j}\right) \\
& \times \sum_{\left(n_{k}\right)}\left(k_{\lambda_{1}} \cdots k_{\lambda_{n_{k}}}\right) \sum_{\left(n_{\bar{k}}\right)}\left(\bar{k}_{\lambda_{n_{k}+1}} \cdots \bar{k}_{\lambda_{n_{k}+n_{\bar{k}}}}\right) e_{\lambda_{n_{k}+n_{\bar{k}}+1}}^{i_{1}} \cdots e_{\lambda_{s-3}-n_{k}-n_{\bar{k}}}^{i_{s-3}} .
\end{aligned}
$$


For $f$ to be traceless, we have thus the additional condition

$$
\begin{aligned}
& \sum_{j=1}^{d-2} f_{\left(n_{k}\right)\left(n_{\bar{k}}\right) i_{1} \ldots i_{s-3-n_{k}-n_{\bar{k}}} j j}=2(k \bar{k}) f_{\left(n_{k}+1\right)\left(n_{\bar{k}}+1\right) i_{1} \ldots i_{s-3-n_{k}-n_{\bar{k}}}} \\
& \forall n_{k} \in\{0, \ldots, s-3\}, \quad n_{\bar{k}} \in\left\{0, \ldots, s-3-n_{k}\right\} .
\end{aligned}
$$

For $n_{\bar{k}} \geq 1$ this becomes (using (A.6))

$$
\begin{aligned}
& \sum_{j=1}^{d-2} X_{\left(n_{k}\right)\left(n_{\bar{k}}-1\right) i_{1} \ldots i_{s-3-n_{k}-n_{\bar{k}}} j j}=2(k \bar{k}) X_{\left(n_{k}+1\right)\left(n_{\bar{k}}\right) i_{1} \ldots i_{s-3-n_{k}-n_{\bar{k}}}} \\
& \forall n_{k} \in\{0, \ldots, s-4\}, \quad n_{\bar{k}} \in\left\{1, \ldots, s-3-n_{k}\right\}
\end{aligned}
$$

and is automatically fulfilled due to the tracelessness of $X$ (i.e. the double tracelessness of the current $J$ ). For $n_{\bar{k}}=0$, however, we get the additional condition

$$
\sum_{j=1}^{d-2} f_{\left(n_{k}\right)(0) i_{1} \ldots i_{s-3-n_{k}} j j}=2 X_{\left(n_{k}+1\right)(0) i_{1} \ldots i_{s-3-n_{k}}} \quad \forall n_{k} \in\{0, \ldots, s-3\} .
$$

At this point it is useful to know the general form of the traceless-projector $\Pi$ given in (C.1). Certainly, the projector obeys $\operatorname{Tr}(A-\Pi A)=\operatorname{Tr} A$ for any dimension and rank and the explicit form of the projector shows that $(A-\Pi A)$ does not contain $A$ itself but is some function $F$ of only $\operatorname{Tr} A$ and higher traces, i.e. $(A-\Pi A)=F(\operatorname{Tr} A)$. One thus can replace $\operatorname{Tr} A$ in $\operatorname{Tr} F(\operatorname{Tr} A)=\operatorname{Tr} A$ by an untraced tensor $B$ and obtains $\operatorname{Tr} F(B)=B$, so that the function $F$ provides a solution for (A.7). In our case we have the effective dimension $d-2($ instead of $d)$ and the rank $s-1-n_{k}$ (instead of $s$ ). The solution for the above equation (A.7) is thus given by

$$
\begin{aligned}
& f_{\left(n_{k}\right)(0) i_{1} \ldots i_{s-1-n_{k}}=}-2 \sum_{l=1}^{\left[\left(s-1-n_{k}\right) / 2\right]} \frac{1}{l !(-2)^{l}} \frac{\left(d / 2+s-l-4-n_{k}\right) !}{\left(d / 2+s-4-n_{k}\right) !} \\
& \times \sum_{2} \delta_{i_{1} i_{2}} \cdots \sum_{2} \delta_{i_{2 l-1}} i_{2 l} \sum_{j_{1}=1}^{d-2} \cdots \sum_{j_{l-1}=1}^{d-2} X_{\left(n_{k}+1\right)(0) j_{1} j_{1} \ldots j_{l-1} j_{l-1} i_{2 l+1} \ldots i_{s-1-n_{k}}} \\
& \forall n_{k} \in\{0, \ldots, s-3\} .
\end{aligned}
$$

Now it remains to extract the expansion coefficients $X_{\left(n_{k}\right)\left(n_{\bar{k}}-1\right) i_{1} \ldots i_{s-1-n_{k}-n_{\bar{k}}}}$ from $X_{\lambda_{1} \ldots \lambda_{s-2}}$ via

$$
X_{\left(n_{k}\right)\left(n_{\bar{k}}-1\right) i_{1} \ldots i_{s-1-n_{k}-n_{\bar{k}}}}=\frac{1}{(k \bar{k})^{n_{k}+n_{\bar{k}}-1}}\left(\bar{k}^{\rho}\right)^{n_{k}}\left(k^{\rho}\right)^{n_{\bar{k}}-1} X_{\rho \ldots \rho i_{1} \ldots i_{s-1-n_{k}-n_{\bar{k}}}} .
$$

Plugging these coefficients into (A.6) and this in turn into the basis-expansion of $f$ yields the final solution of (A.5) or (A.1)

$$
\begin{aligned}
f_{\lambda_{1} \ldots \lambda_{s-1}}= & \sum_{n_{k}=0}^{s-2} \sum_{n_{\bar{k}}=1}^{s-1-n_{k}} \frac{1}{(k \bar{k})^{n_{k}+n_{\bar{k}}}} \bar{k}^{\rho_{1}} \cdots \bar{k}^{\rho_{n_{k}}} k^{\rho_{n_{k}+1}} \cdots k^{\rho_{n_{k}+n_{\bar{k}}-1}} X_{\rho_{1} \ldots \rho_{n_{k}+n_{\bar{k}}-1} i_{1} \ldots i_{s-1-n_{k}-n_{\bar{k}}}} \\
& \times \sum_{\left(n_{k}\right)}\left(k_{\lambda_{1}} \cdots k_{\lambda_{n_{k}}}\right) \sum_{\left(n_{\bar{k}}\right)}\left(\bar{k}_{\lambda_{n_{k}+1} \ldots \lambda_{n_{k}+n_{\bar{k}}}}\right)\left(e_{\left.\lambda_{n_{k}+n_{\bar{k}}+1}^{i_{1}} \cdots e_{\lambda_{s-1}}^{i_{s-1-n_{k}-n_{\bar{k}}}}\right)}^{s-1} f_{\left(n_{k}\right)(0) i_{1} \ldots i_{s-1-n_{k}}} \sum_{\left(n_{k}\right)}\left(k_{\lambda_{1}} \cdots k_{\lambda_{n_{k}}}\right)\left(e_{\lambda_{n_{k}+1}}^{i_{1}} \cdots e_{\lambda_{s-1}}^{i_{s-1-n_{k}}}\right),\right.
\end{aligned}
$$


where (due to (A.8) and (A.9)) some coefficients of the last row are given by

$$
\begin{aligned}
& f_{\left(n_{k}\right)(0) i_{1} \ldots i_{s-1-n_{k}}=}-\frac{2}{(k \bar{k})^{n_{k}+1}} \bar{k}^{\rho_{1}} \ldots \bar{k}^{\rho_{n_{k}+1}} \sum_{l=1}^{\left[\left(s-1-n_{k}\right) / 2\right]} \frac{1}{l !(-2)^{l}} \frac{\left(d / 2+s-l-4-n_{k}\right) !}{\left(d / 2+s-4-n_{k}\right) !} \\
& \times \sum_{2} \delta_{i_{1} i_{2}} \ldots \sum_{2} \delta_{i_{2 l-1} i_{2 l}} \sum_{j_{1}=1}^{d-2} \ldots \sum_{j_{l-1}=1}^{d-2} X_{\rho_{1} \ldots \rho_{n_{k}+1} j_{1} j_{1} \ldots j_{l-1} j_{l-1} i_{2 l+1} \ldots i_{s-1-n_{k}}} \\
& \forall n_{k} \in\{0, \ldots, s-3\} .
\end{aligned}
$$

Note that the coefficients $f_{(s-2)(0) i_{1} \ldots i_{s-1-n_{k}}}$ and $f_{(s-1)(0) i_{1} \ldots i_{s-1-n_{k}}}$ are still undetermined and can be chosen arbitrarily.

For $s=3$ we have

$$
f_{(0)(0) i_{1} i_{2}}=\frac{2}{(d-2)(k \bar{k})} \bar{k}^{\rho} \delta_{i_{1} i_{2}} X_{\rho}
$$

and the solution becomes

$$
\begin{aligned}
f_{\lambda_{1} \lambda_{2}}= & \frac{1}{k \bar{k}} X_{i_{1}} \sum_{1} \bar{k}_{\lambda_{1}} e_{\lambda_{2}}^{i_{1}}+\frac{1}{(k \bar{k})^{2}} k^{\rho} X_{\rho} \sum_{2} \bar{k}_{\lambda_{1}} \bar{k}_{\lambda_{2}}+\frac{1}{(k \bar{k})^{2}} \bar{k}^{\rho} X_{\rho} \sum_{1} k_{\lambda_{1}} \bar{k}_{\lambda_{2}} \\
& +\frac{2}{(d-2) k \bar{k}} \bar{k}^{\rho} X_{\rho} \sum_{i=1}^{d-2} e_{\lambda_{1}}^{i} e_{\lambda_{2}}^{i}+f_{(1)(0) i_{1}} \sum_{1} k_{\lambda_{1}} e_{\lambda_{2}}^{i_{1}}+f_{(2)(0)} k_{\lambda_{1}} k_{\lambda_{2}} .
\end{aligned}
$$

For an appropriate choice of $f_{(1)(0) i_{1}}$ and $f_{(2)(0)}$ this coincides in fact with the solution (A.2) given in the beginning, if the metric $\eta^{\mu \nu}$ in (A.2) is expanded as in (4.8).

\section{B Symmetrization}

For the tensor product of two symmetric tensors it is very convenient to introduce the symmetrized sums which run over those permutations of uncontracted indices which lead to inequivalent terms. For example

$$
\begin{aligned}
& \sum_{1} A_{\lambda_{1}} B_{\lambda_{2} \ldots \lambda_{s}} \equiv A_{\lambda_{1}} B_{\lambda_{2} \ldots \lambda_{s}}+\underset{\substack{\lambda_{2} \\
s \text { terms }}}{B_{\lambda_{1} \lambda_{3} \ldots \lambda_{s}}+\cdots+A_{\lambda_{s}} B_{\lambda_{1} \ldots \lambda_{s}-1}}, \\
& \sum_{2} A_{\lambda_{1} \lambda_{2}} B_{\lambda_{3} \ldots \lambda_{s}} \equiv \sum_{i<j} A_{\lambda_{i} \lambda_{j}} B_{\lambda_{1} \ldots \lambda_{i-1} \lambda_{i+1} \ldots \lambda_{j-1} \lambda_{j+1} \ldots \lambda_{s}} . \\
& s(s-1) / 2 \text { terms }
\end{aligned}
$$

This differs from the projection to the symmetric part (denoted by a round bracket around the indices) only by a normalization factor. In the above cases we have $A_{\left(\lambda_{1}\right.} B_{\left.\lambda_{2} \ldots \lambda_{s}\right)}=\frac{1}{s} \sum_{1} A_{\lambda_{1}} B_{\lambda_{2} \ldots \lambda_{s}}$ and $A_{\left(\lambda_{1} \lambda_{2}\right.} B_{\left.\lambda_{3} \ldots \lambda_{s}\right)}=\frac{2}{s(s-1)} \sum_{2} A_{\lambda_{1} \lambda_{2}} B_{\lambda_{2} \ldots \lambda_{s}}$. In general the projection to the symmetric part is given by

$$
X_{\left(\lambda_{1} \ldots \lambda_{s}\right)} \equiv \frac{1}{s !} \sum_{\text {all Perm's } P} X_{\lambda_{P(1)} \ldots \lambda_{P(s)}} .
$$

It has the projection-property $X_{\left(\left(\lambda_{1} \ldots \lambda_{s}\right)\right)}=X_{\left(\lambda_{1} \ldots \lambda_{s}\right)}$ and appears automatically when $X$ is contracted with any other symmetric tensor, in particular with the $s$-th power of a vector $X_{\lambda_{1} \ldots \lambda_{s}} v^{\lambda_{1}} \cdots v^{\lambda_{s}}=X_{\left(\lambda_{1} \ldots \lambda_{s}\right)} v^{\lambda_{1}} \cdots v^{\lambda_{s}}$.

The different normalization in the symmetrized sums (B.1) and (B.2) is in turn more convenient in calculations. To study the general properties of theses symmetrized sums, let us first 
note that one can extend the definition to a product of a symmetric rank $p$ and a rank $q$ tensor. It can be defined as

$$
\begin{aligned}
\sum_{(p)} A_{\lambda_{1} \ldots \lambda_{p}}^{(p)} B_{\lambda_{p+1} \ldots \lambda_{p+q}}^{(q)} & \equiv\left(\begin{array}{c}
p+q \\
p
\end{array}\right) A_{\left(\lambda_{1} \ldots \lambda_{p}\right.}^{(p)} B_{\left.\lambda_{p+1} \ldots \lambda_{p+q}\right)}^{(q)} \\
& =\frac{1}{p ! q !} \sum_{\text {all Perm's } P} A_{\lambda_{P(1) \ldots \lambda_{P(p)}}^{(p)}}^{(p)} B_{\lambda_{P(p+1)} \ldots \lambda_{P(s)}}^{(q)} .
\end{aligned}
$$

We have put the $p$ below the sum in brackets in order to avoid confusion with a sum over all $p$. Instead $p$ is fixed here. Again this symmetrized sum can be understood as the sum over all $\left(\begin{array}{c}p+q \\ p\end{array}\right)$ inequivalent terms. Because of $\left(\begin{array}{c}p+q \\ p\end{array}\right)\left(\begin{array}{c}p+q+r \\ p+q\end{array}\right)=\left(\begin{array}{c}p+q+r \\ p\end{array}\right)\left(\begin{array}{c}q+r \\ q\end{array}\right)=\frac{(p+q+r) !}{p ! q ! r !}$, this symmetrized sum is associative in the sense

$$
\sum_{(p+q)}\left(\sum_{(p)} A^{(p)} B^{(q)}\right) C^{(r)}=\sum_{(p)} A^{(p)}\left(\sum_{(q)} B^{(q)} C^{(r)}\right) .
$$

In addition it has a Leibniz-like behaviour with respect to index contractions. If we denote the result of (B.3) by

$$
C_{\lambda_{1} \ldots \lambda_{p+q}}^{(p+q)} \equiv \sum_{(p)} A_{\lambda_{1} \ldots \lambda_{p}}^{(p)} B_{\lambda_{p+1} \ldots \lambda_{p+q}}^{(q)}
$$

then the contraction with a vector acts like a derivative

$$
v^{\mu} C_{\mu \lambda_{2} \ldots \lambda_{p+q}}^{(p+q)}=\sum_{(p-1)} v^{\mu} A_{\mu \lambda_{2} \ldots \lambda_{p}}^{(p)} B_{\lambda_{p+1} \ldots \lambda_{p+q}}^{(q)}+\sum_{(p)} A_{\lambda_{2} \ldots \lambda_{p+1}}^{(p)} v^{\mu} B_{\mu \lambda_{p+2} \ldots \lambda_{p+q}}^{(q)} .
$$

Contractions with rank $r$ tensors act like derivatives of order $r$

$$
\begin{aligned}
D_{(r)}^{\mu_{1} \ldots \mu_{r}} C_{\mu_{1} \ldots \mu_{r} \lambda_{1} \ldots \lambda_{p+q-r}}^{(p+q)}= & \sum_{(p-r)} A_{\lambda_{1} \ldots \lambda_{p-r} \mu_{1} \ldots \mu_{r}}^{(p)} D_{(r)}^{\mu_{1} \ldots \mu_{r}} B_{\lambda_{p-r+1} \ldots \lambda_{p+q-r}}^{(q)} \\
& +\sum_{(p-r+1)} r A_{\lambda_{1} \ldots \lambda_{p-r+1} \mu_{1} \ldots \mu_{r-1}}^{(p)} D_{(r)}^{\mu_{1} \ldots \mu_{r}} B_{\mu_{r} \lambda_{p-r+2} \ldots \lambda_{p+q}-r}^{(q)}+\cdots \\
& +\sum_{(p-i)}\left(\begin{array}{c}
r \\
i
\end{array}\right) A_{\lambda_{1} \ldots \lambda_{p-i} \mu_{1} \ldots \mu_{i}}^{(p)} D_{(r)}^{\mu_{1} \ldots \mu_{r}} B_{\mu_{i+1} \ldots \mu_{r} \lambda_{p-i+1} \ldots \lambda_{p+q-r}}^{(q)}+\cdots \\
& +\sum_{(p)} A_{\lambda_{1} \ldots \lambda_{p}}^{(p)} D_{(r)}^{\mu_{1} \ldots \mu_{r}} B_{\mu_{1} \ldots \mu_{r} \lambda_{p+1} \ldots \lambda_{p+q-r}}^{(q)} .
\end{aligned}
$$

This behaviour is very convenient for calculating divergencies (contracting with $k^{\mu}$ ) or traces (contracting with the metric $\eta^{\mu \nu}$ ).

\section{Projection to the (double) traceless part}

For higher rank symmetric tensors the trace is defined by contracting any pair of indices with the metric and will be denoted by

$$
A_{\lambda_{3} \ldots \lambda_{s}}^{\prime} \equiv \eta^{\lambda_{1} \lambda_{2}} A_{\lambda_{1} \lambda_{2} \lambda_{3} \ldots \lambda_{s}}
$$

The projector $\Pi_{\lambda_{1} \ldots \lambda_{s}} \rho_{1} \ldots \rho_{s}$ to the traceless part of an arbitrary symmetric rank $s$ tensor field $A_{\lambda_{1} \ldots \lambda_{s}}$ is then given by

$$
(\Pi A)_{\lambda_{1} \ldots \lambda_{s}} \equiv A_{\lambda_{1} \ldots \lambda_{s}}-\frac{1}{d+2 s-4} \sum_{2} \eta_{\lambda_{1} \lambda_{2}} A_{\lambda_{3} \ldots \lambda_{s}}^{\prime}
$$




$$
+\sum_{k=2}^{[s / 2]} \frac{1}{k !(-2)^{k}} \frac{(d / 2+s-k-2) !}{(d / 2+s-2) !} \underbrace{\sum_{2} \eta_{\lambda_{1} \lambda_{2}} \cdots \sum_{2} \eta_{\lambda_{2 k-1} \lambda_{2 k}} A_{\lambda_{2 k+1} \ldots \lambda_{s}}^{\left({ }^{\prime} k\right)}}_{\frac{s !}{2^{k}(s-2 k) !} \text { terms }}
$$

where $A^{\left({ }^{\prime} k\right)}$ is the $k$-th trace of $A,[s / 2] \equiv\left\{\begin{array}{l}s / 2 \text { for } s \text { even } \\ (s-1) / 2 \text { for } s \text { odd }\end{array}\right.$ is the integer part of $s / 2$ and the factorials have to be understood via the $\Gamma$-function in the case of an odd dimension $d$. One can convince oneself that $(\Pi A)^{\prime}=0$ for any $A$. When $A$ is double traceless $\left(A^{\prime \prime}=0\right)$, only the first line contributes to the projection. When $A$ is already traceless, the projector reduces to the identity as it should. One could define various different projections to the subspace of traceless tensors. The important property of this one is the fact that the operator is symmetric in the sense $\Pi_{\lambda_{1} \ldots \lambda_{s}}{ }^{\rho_{1} \ldots \rho_{s}}=\Pi_{{ }_{1} \ldots \rho_{s}}^{\rho_{1} \ldots \lambda_{s}}$. The existence of a symmetric projection operator to some subspace always guarantees that tensors contracted with some subspace element get projected to the same subspace. If we contract for example the traceless tensor $(\Pi A)_{\lambda_{1} \ldots \lambda_{s}}$ with an arbitray tensor $B_{\lambda_{1} \ldots \lambda_{s}}$, we can write $(\Pi A)_{\lambda_{1} \ldots \lambda_{s}} B^{\lambda_{1} \ldots \lambda_{s}}=A_{\lambda_{1} \ldots \lambda_{s}}(\Pi B)^{\lambda_{1} \ldots \lambda_{s}}$.

Similarly to above, one can project to a double traceless part, where only double and higher traces are subtracted appropriately

$$
\left(\Pi^{(d t l)} A\right)_{\lambda_{1} \ldots \lambda_{s}} \equiv A_{\lambda_{1} \ldots \lambda_{s}}-\sum_{k=2}^{[s / 2]} \frac{k-1}{(-2)^{k} k !} \cdot \frac{(d / 2+s-k-3) !}{(d / 2+s-3) !} \underbrace{\sum_{2} \eta_{\lambda_{1} \lambda_{2}} \ldots \sum_{2} \eta_{\lambda_{2 k-1} \lambda_{2 k}} A_{\lambda_{2 k+1} \ldots \lambda_{s}}^{\left({ }^{\prime} k\right)}}_{\frac{s !}{2^{k}(s-2 k) !} \text { terms }} .
$$

Again one can check that indeed $\left(\Pi^{(d t l)} A\right)^{\prime \prime}=0$ for all symmetric rank $s$ tensor fields $A$. We will not make use of this projection operator in the main text, but it is important to keep in mind that this projector would have been needed, if the variation of the Fronsdal action (2.1) with respect to the (double traceless) tensor gauge field did not automatically produce a double traceless expression (2.6). Note finally that also this projector acts symmetrically. This is the reason why the double traceless property of the current $J$ is inherited by the contraction with the double traceless tensor gauge field $A$ in the action (2.1).

\section{Acknowledgements}

The work of S.G. was supported by ENRAGE (European Network on Random Geometry), a Marie Curie Research Training Network, contract MRTN-CT-2004-005616. The work of G.S. was partially supported by the EEC Grant no. MRTN-CT-2004-005616.

\section{References}

[1] Schwinger J., Particles, sources, and fields, Addison-Wesley, Reading, MA, 1970.

[2] Fronsdal C., Massless fields with integer spin, Phys. Rev. D 18 (1978), 3624-3629.

[3] Singh L.P.S., Hagen C.R., Lagrangian formulation for arbitrary spin. I. The boson case, Phys. Rev. D 9 (1974), 898-909.

[4] Feynman R.P., Feynman lecture on gravitation, Westview Press, 2002.

[5] Fierz M., Über die relativistische Theorie kräftefreier Teilchen mit beliebigem Spin, Helv. Phys. Acta. 12 (1939), 3-27.

[6] Chang S.J., Lagrange formulation for systems with higher spin, Phys. Rev. 161 (1967), 1308-1315.

[7] van Dam H., Veltman M.J.G., Massive and massless Yang-Mills and gravitational fields, Nuclear Phys. B 22 (1970), 397-411. 
[8] Curtright T., Massless field supermultiplets with arbitrary spin, Phys. Lett. B 85 (1979), 219-224.

[9] Bengtsson A.K., Bengtsson I., Brink L., Cubic interaction terms for arbitrary spin, Nuclear Phys. B 227 (1983), 31-40.

[10] Bengtsson A.K., Bengtsson I., Brink L., Cubic interaction terms for arbitrarily extended supermultiplets, Nuclear Phys. B 227 (1983), 41-49.

[11] Bengtsson A.K.H., Bengtsson I., Linden N., Interacting higher spin gauge fields on the light front, Classical Quantum Gravity 4 (1987), 1333-1345.

[12] Berends F.A., Burgers G.J.H., van Dam H., On the theoretical problems in constructing interactions involving higher-spin massless particles, Nuclear Phys. B 260 (1985), 295-322.

[13] Berends F.A., Burgers G.J.H., van Dam H., On spin three selfinteractions, Z. Phys. C 24 (1984), $247-254$.

[14] de Wit B., Freedman D.Z., Systematics of higher spin gauge fields, Phys. Rev. D (3) 21 (1980), $358-367$.

[15] Bengtsson A.K.H., Structure of higher spin gauge interactions, J. Math. Phys. 48 (2007) 072302, 35 pages, hep-th/0611067.

[16] Savvidy G., Non-Abelian tensor gauge fields: enhanced symmetries, hep-th/0604118 (see Section 6).

[17] Bengtsson A.K.H., Towards unifying structures in higher spin gauge symmetry, SIGMA 4 (2008), 013, 23 pages, arXiv:0802.0479.

[18] Bekaert X., Cnockaert S., Iazeolla C., Vasiliev M.A., Nonlinear higher spin theories in various dimensions, hep-th/0503128.

[19] Vasiliev M.A., Higher-spin gauge theories in four, three and two dimensions, Internat. J. Modern Phys. D 5 (1996), 763-797, hep-th/9611024.

[20] Vasiliev M.A., Higher spin gauge theories: star-product and AdS space, hep-th/9910096.

[21] Engquist J., Sezgin E., Sundell P., On $N=1,2,4$ higher spin gauge theories in four dimensions, Classical Quantum Gravity 19 (2002), 6175-6196, hep-th/0207101.

[22] Sezgin E., Sundell P., Holography in 4D (super) higher spin theories and a test via cubic scalar couplings, J. High Energy Phys. 2005 (2005), no. 7, 044, 26 pages, hep-th/0305040.

[23] Francia D., Sagnotti A., On the geometry of higher-spin gauge fields, Classical Quantum Gravity 20 (2003), S473-S486, hep-th/0212185.

[24] Francia D., Mourad J., Sagnotti A., Current exchanges and unconstrained higher spins, Nuclear Phys. B 773 (2007), 203-237, hep-th/0701163.

[25] Sorokin D., Introduction to the classical theory of higher spins, AIP Conf. Proc. 767 (2005), 172-202, hep-th/0405069.

[26] Francia D., Sagnotti A., Free geometric equations for higher spins, Phys. Lett. B 543 (2002), 303-310, hep-th/0207002.

[27] Savvidy G., Non-Abelian tensor gauge fields: generalization of Yang-Mills theory, Phys. Lett. B 625 (2005), 341-350, hep-th/0509049.

[28] Savvidy G., Non-Abelian tensor gauge fields. I, Internat. J. Modern Phys. A 21 (2006), 4931-4957.

[29] Savvidy G., Non-Abelian tensor gauge fields. II, Internat. J. Modern Phys. A 21 (2006), 4959-4977.

[30] Barrett J. K., Savvidy G., A dual Lagrangian for non-Abelian tensor gauge fields, Phys. Lett. B 652 (2007), 141-145, arXiv:0704.3164

[31] Weinberg S., Feynman rules for any spin, Phys. Rev. (2) 133 (1964), B1318-B1332.

[32] Weinberg S., The quantum theory of fields, Vol. 1, Foundations, Cambridge University Press, Cambridge, 1995.

[33] Konitopoulos S., Savvidy G., Production of spin-two gauge bosons, arXiv:0804.0847. 